Title : will be set by the publisher

Editors : will be set by the publisher

EAS Publications Series, Vol. ?, 2018

\title{
EARTHSHINE OBSERVATIONS OF AN INHABITED PLANET
}

\author{
Enric Pallé ${ }^{1}$
}

\begin{abstract}
Earthshine is sunlight that has been reflected from the dayside Earth onto the dark side of the Moon and back again to Earth. In recent times, there has been renewed interest in ground-based visible and near-infrared measurements of earthshine as a proxy for exoplanet observations. Observations of earthshine allow us to explore and characterize the globally integrated photometric, spectral and polarimetric features of the Earth, and to extract precise information on the distinctive characteristics of our planet, and life in particular. They also allow us to quantify how this feature changes with time and orbital configuration. Here we present a brief review of the main earthshine observations and results.
\end{abstract}

\section{Introduction}

Over the past few years, we have developed the capacity to discover planets orbiting around stars other than the Sun, and the number of detections is increasing exponentially. Even though we are not yet capable of detecting and exploring Earthlike planets, ambitious missions are already being planned for the coming decades. In the near future, it is likely that Earth-size planets will be discovered, and efforts will then be directed towards obtaining images and spectra from them.

The resulting spatially integrated observations will need to be compared to observations of similar resolution of the only planet that we know to be inhabited, namely Earth. The scientific and technological advances of the XXth century have brought a finer and finer detailed knowledge of our planet. Routinely, plankton blooms, city night lights or crop health indices are measured from space. However, when observing an extrasolar planet, all the reflected starlight and the radiated emission from its surface and atmosphere will be integrated into a single point, and most of these features will disappear in the noise when global averages are taken.

Despite the fact that Earth is our own planet, observations from a remote perspective are limited. They can only be obtained from a very remote observing

\footnotetext{
${ }^{1}$ Instituto de Astrofisica de Canarias, Via Lactea s/n, 38205 La Laguna, Tenerife, Spain
} 
Title : will be set by the publisher

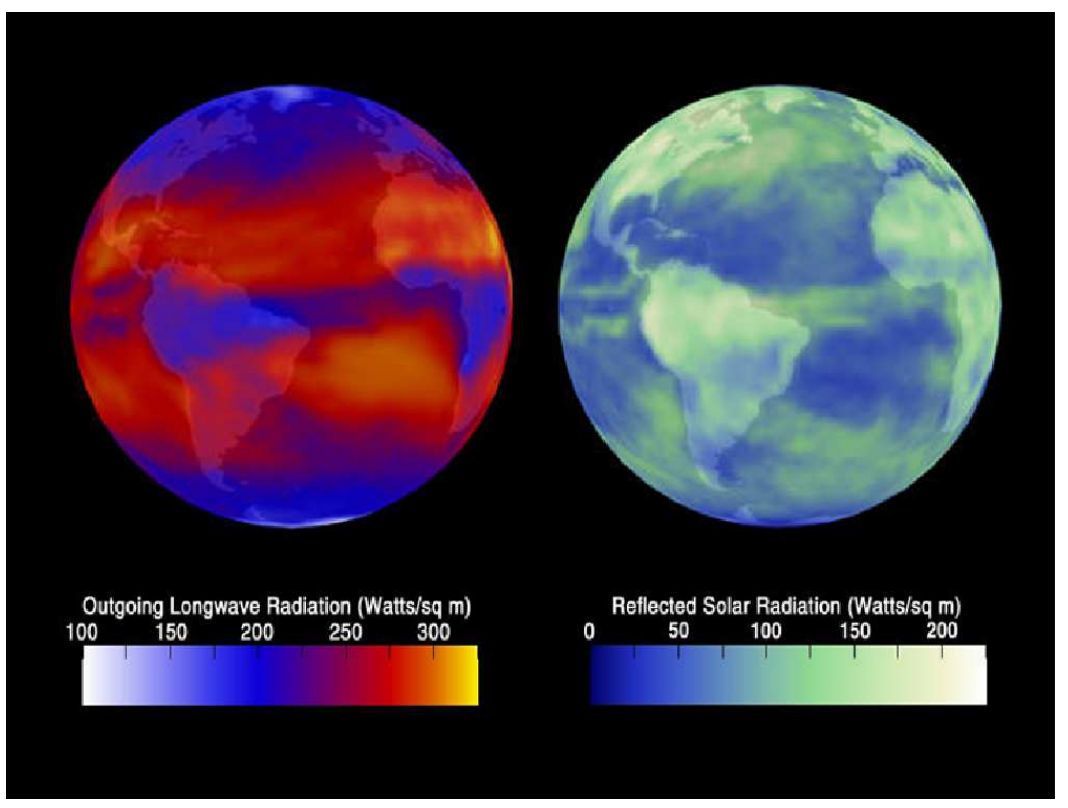

Fig. 1. The spatial distribution of the thermal radiation emitted into space from the Earth's surface and atmosphere (left), and the sunlight reflected back into space by oceans, land, aerosols and clouds (right). Image credit: NASA

platform, from a complex calibration of low Earth orbit satellite observations, or from the ground by observing the earthshine reflected on the dark side of the Moon.

What these observations reveal about our planet will be highly dependent on several factors. The observing geometry will determine whether one samples either the full surface of the planet or just one of the hemispheres. The spectral range is also important, as the light reflected or emitted by Earth is not uniformly distributed (see Figure 11). Finally, timing is also important. Our planet has undergone evolution with time, so an external observer would obtain a different view of Earth if observing it at different epochs (Kaltenegger et al. 2007). In this paper we concentrate on modern Earth observations, mostly in the visible range, as obtained from earthshine observations.

\section{The Earthshine}

Earthshine is sunlight reflected from the dayside Earth onto the dark side of the Moon and reflected back again to Earth. The term "dark side" refers to the portion of the lunar surface that, at any instant, faces the Earth but not the Sun. Both earthshine, from the dark side of the moon, and moonlight, from the bright side of 

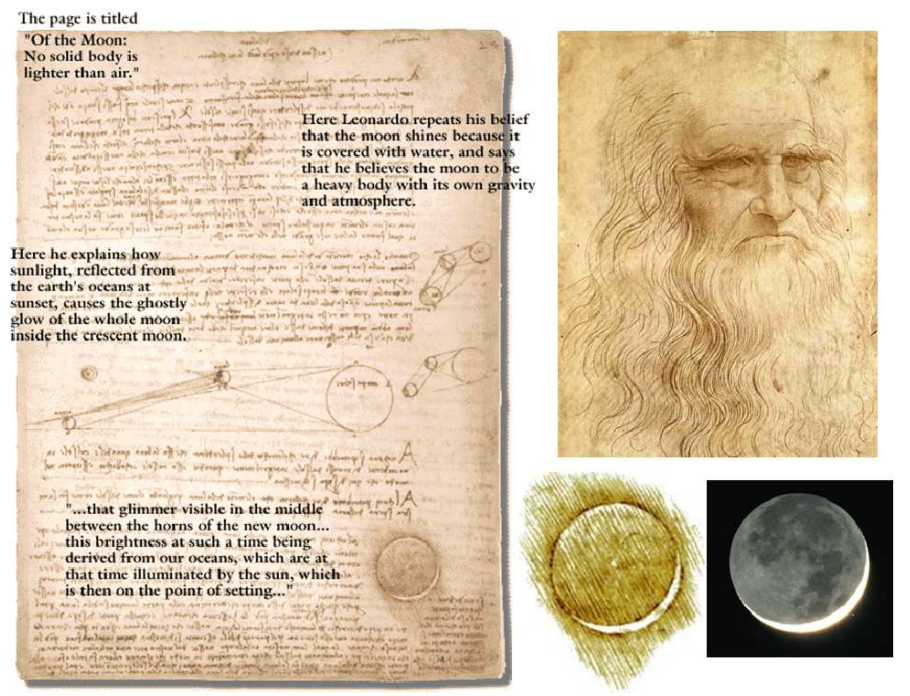

Fig. 2. Reproduction of a page of Leonardo da Vinci's Codex Leicester. Also shown is a image of da Vinci in his Autoportrait and a comparison of a real picture of the earthshine with one of his drawings. Image credit: American Museum of Natural History Library

the moon, are transmitted through the same airmass just prior to detection and thus suffer the same extinction and imposed absorption features. Their ratio is the averaged reflection coefficient (or albedo) of the global atmosphere, the global atmosphere being defined as the portion of dayside Earth simultaneously visible from the Sun and the Moon.

Already in the XVIth century, Leonardo da Vinci had correctly deduced the nature of the Earthshine. In his Codex Leicester (circa 1510), he states his belief that the Moon possessed an atmosphere and oceans, and that it was a fine reflector of light because it was covered with so much water. He also speculated about how storms on Earth could cause the Earthshine to become brighter or dimmer, which is indeed observable with modern instrumentation. After Leonardo, others (such as Galileo) continued to observe the earthshine in historical times. In recent times, however, there has been a renewed interest in ground-based visible and nearinfrared measurements of the earthshine as a proxy for exoplanet observations (Woolf et al. 2002), and also for climate studies (Pallé et al. 2004).

\section{Earth's reflected light}

The light reflected by the Earth in the direction of a hypothetical distant observer will change in time, depending on the orbital phase, rotation, seasonality (tilt angle) and weather patterns. Each observing perspective of Earth will have its unique features and will present different photometric variability. Figure 3 illus- 
Title : will be set by the publisher

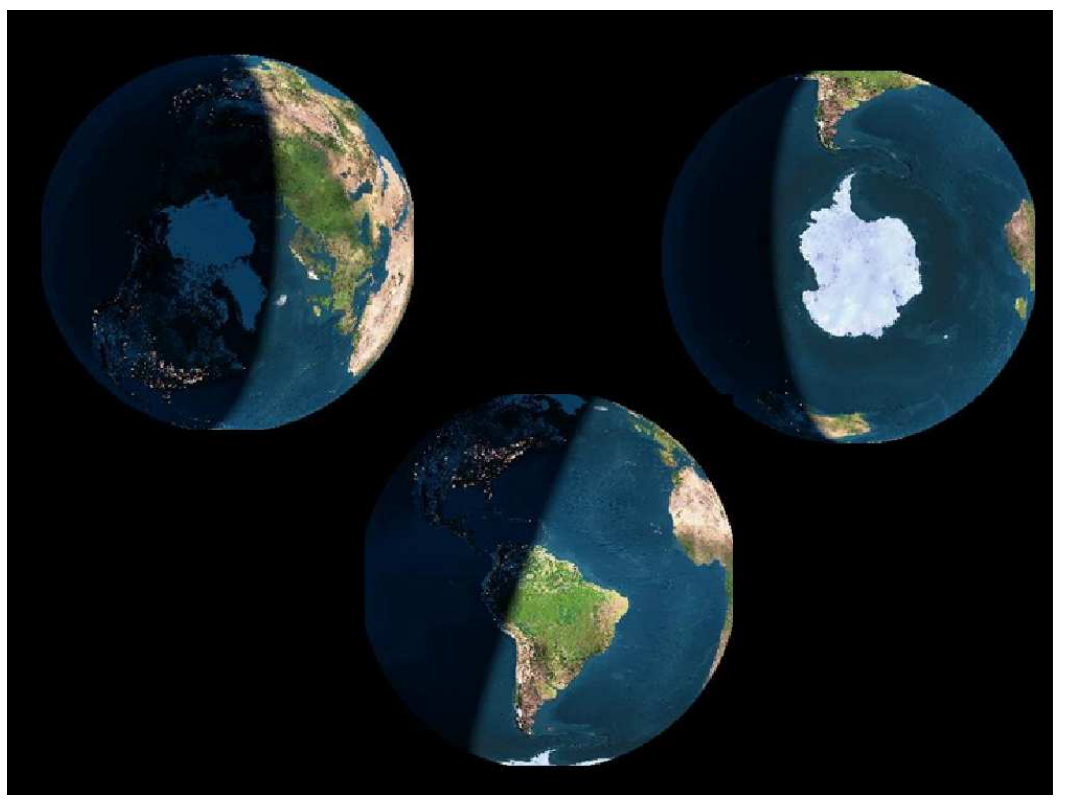

Fig. 3. The Earth from different viewpoints. The three images show the Earth for the exact same date and time (2003/11/19 at 10:00 UT) but from three different perspectives: from $90^{\circ}$ above the ecliptic (north polar view) (right), from $90^{\circ}$ below the ecliptic (south polar view) (left), and from within the ecliptic plane (centre).

trates this point: Several views of Earth are represented for the exact same date and time, but judging from the visible scenery features, the three images could well represent three different planets. Note, however, that the figures are misleading in the sense that clouds are missing.

A number of earthshine measurements have documented changes with phase and rotation but are necessarily limited in their range of observations. From these measurements the photometric variability of the Earth is found to be of the order of 10-15\% from night to night during one Earth rotation (Qiu et al. 2003). For an observer outside the solar system, Earth observations will differ from the mostly equatorial perspective offered by the earthshine measurements, or the global averages obtained from polar orbiter satellites. The total reflected flux in a given direction, $\beta$, can be calculate using

$$
F_{e}(\beta)=S \pi R_{e}^{2} p_{e} f_{e}(\beta),
$$

where $S$ is the solar flux at the top of the Earth's atmosphere $\left(1370 \mathrm{~W} / \mathrm{m}^{2}\right)$, and $R_{e}, p_{e}$, and $f_{e}(\beta)$ are the radius, geometric albedo and phase function of the Earth, respectively. There is a systematic variation of $p_{e} f_{e}(\beta)$ throughout the Earth's orbital period (sidereal year), and fluctuations of $p_{e} f_{e}(\beta)$ about its sys- 
tematic behaviour are caused by varying terrestrial conditions, including weather and seasons (Pallé et al. 2004).

Among other physical properties, the identification of the rotation rate of an exoplanet with relative accuracy will be important for several reasons. If the rotation period of an Earthlike planet can be determined accurately, one can then fold the photometric light curves at the rotation period to study regional properties of the planet's surface and/or atmosphere. With phased light curves it could be possible study local surface or atmospheric properties with follow-up photometry, spectroscopy and polarimetry to detect surface and atmospheric inhomogeneities and to improve the sensitivity to localized biomarkers. Exoplanets, however, are expected to deviate widely in their physical characteristics: if they have no strong surface features (Mercury or Mars), or they are completely covered by clouds (Venus and the giant planets), determining the rotational period may be an impossible task.

Palle et al. (2008) found that scattered light observations of the Earth could accurately identify the rotation period of the Earth's surface. This is because large-scale time averaged cloud patterns are tied to the surface features of Earth, such as continents and ocean currents. This relatively fixed nature of clouds is the key point that would allow Earth's rotation period to be determined from afar. The lifetime of large-scale cloud systems on Earth is typically about one to two weeks (roughly 10 times the rotational period). In fact, Earth may well be the only one of the major planets for which a rotational period can be easily established from a distance of several AU.

\subsection{Terrestrial clouds}

Clouds are common on solar system planets, and even on satellites with dense atmospheres. On Earth, clouds are continuously forming and disappearing, covering an average of about $60 \%$ of the Earth's surface (Rossow et al. 1996). This feature is unique in the solar system to Earth: only the Earth has large-scale cloud patterns that partially cover the planet and partly reveal the rocky surface, and change on timescales comparable to the rotational period. This is because the

temperature and pressure on the Earth's surface allow for water to change phase with relative ease from solid to liquid to gas.

Pallé et al. (2008) studied the periodicity shifts that appear in modelled Earth observations (see Figure 44), and concluded that they are introduced by the largescale wind and cloud patterns. As these patterns change, the apparent rotational period of the Earth is sometimes shorter than 24 hours. Thus, for an extrasolar Earth-like planet, photometric observations could be used to infer the presence of a "variable" surface (i.e. clouds), even in the absence of spectroscopic data. This would strongly suggest the presence of liquid water on the planet's surface, especially if the effective temperature of the planet was also determined by other means. 


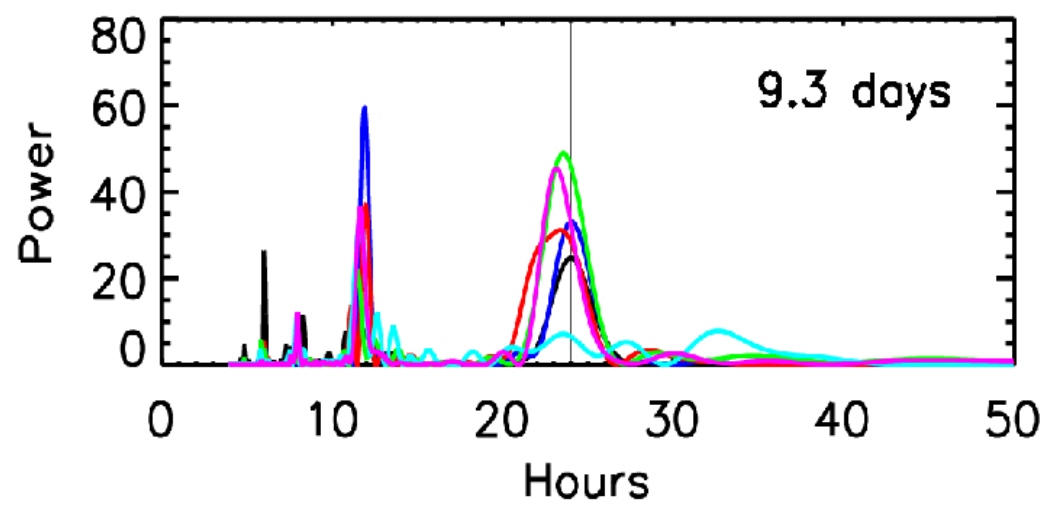

Fig. 4. Periodogram analysis of the Earth's scattered light from an equatorial viewing angle. Two months of reflected light simulations are subdivided in six equally long time series, and a periodogram analysis is performed on each series. In the figure, different colours indicate different data subperiods. Note the appreciable decrease in the retrieved rotation rate for some of the time series. Other peaks in the periodogram indicate shorter periodicities introduced by the Earth's land/ocean distribution. Adapted from Pallé et al. (2008).

\subsection{Glint}

Although the reflectivity of water is very low at high and medium angles of incident light, it increases tremendously at small angles of incident sunlight. This is observable from space on the sun-illuminated side of the Earth near the terminator, and it it known as the glint (Campbell et al. 2003). Waviness, however, causes an appreciable reduction in the glint magnitude.

To assess the detectability of specular reflection from an ocean in the (diskaveraged) reflected light from a distant planet, Williams \& Gaidos (2008) used a model to simulate the orbital brightness variation, or "light curve", of Earth. They concluded that ocean-bearing planets with edge-on orbital geometries will exhibit large changes in their apparent reflectivity due to specular reflection. Similar phase brightening of the crescent Earth have been empirically identified in earthshine data (Pallé et al. 2003). Earthshine has also been found to be strongly polarized, which is further indication of specular reflection of sunlight off the oceans (Stam et al. 2006).

\section{Earth's spectral signatures}

Hitchcock \& Lovelock (1967) pointed out that the remote detection of life forms might be possible by studying the atmospheric composition of a planet. On Earth, 


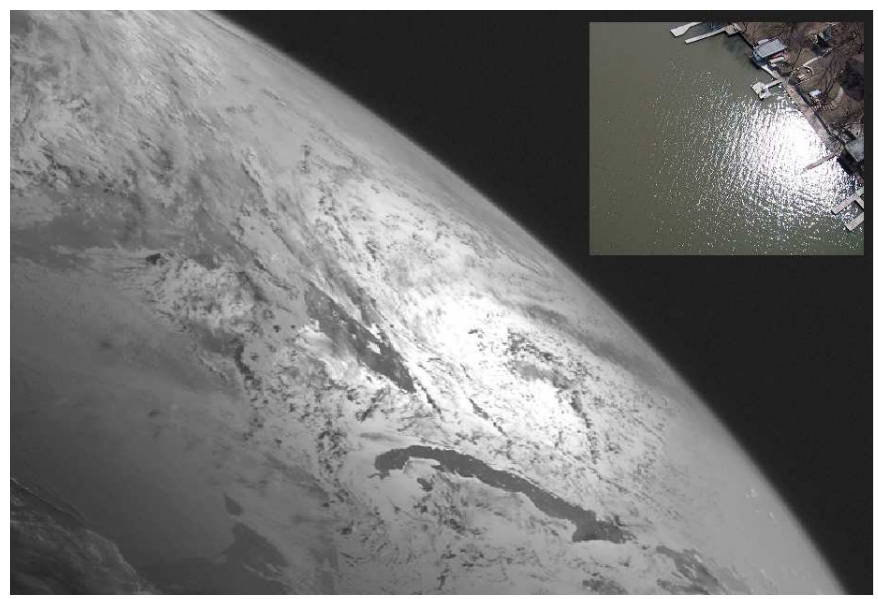

Fig. 5. Sun-glint in the Sargasso Sea, obtained by GOES-West, on 2000 June 22. The top corner illustrates the glint phenomenon at a smaller scale. Image credit: NASA

the presence of $\mathrm{H}_{2} \mathrm{O}, \mathrm{O}_{2}$ and $\mathrm{O}_{3}$ in its atmosphere is clearly marked in the spectra. This particular combination of atmospheric constituents, because they are in disequilibrium, strongly signal the presence of life, the driver of the disequilibrium.

In the past, there have been numerous attempts to observe the spectrum of the pale blued dot from a distance in different spectral regions. Among other efforts, observations of the Earth as a planet from afar have been made in the visible range from the Galileo spacecraft (Sagan et al. 1993).

With the aim of improving the spectral resolution and the sampling of seasonality and phase changes, spectroscopic measurements of the earthshine have also been taken from ground-based observatories and analysed at visible wavelengths (Woolf et al. 2002); Montañés-Rodríguez et al. 2005 and 2006), and in the near infrared wavelengths (Turnbull et al. 2006).

The overall shape of the Earth's spectrum in the visible region shows interesting and peculiar signatures. The most prominent is the Rayleigh scattering, the main source of opacity in the Earth's atmosphere, which shows an enhancement in reflectivity towards the blue part of the spectra (Figure 6). The strong wavelength dependence of the scattering $\left(\lambda^{-4}\right)$ means that blue light is scattered much more than red light. The Rayleigh scattering of sunlight by the Earth's atmosphere is the main reason why the sky is blue, and our planet is known as the blue planet. Except for Neptune and Uranus, no other solar system body shows this strong Rayleigh feature in the blue. However, Neptune and Uranus' predominant blue colour does not come from Rayleigh scattered light, but from the absorption of red and infrared light by methane gas in their atmospheres.

At shorter wavelengths $(<310 \mathrm{~nm})$ ozone absorption dominates over the Rayleigh scattering, causing a strong decrease in reflectance, which has also been measured 


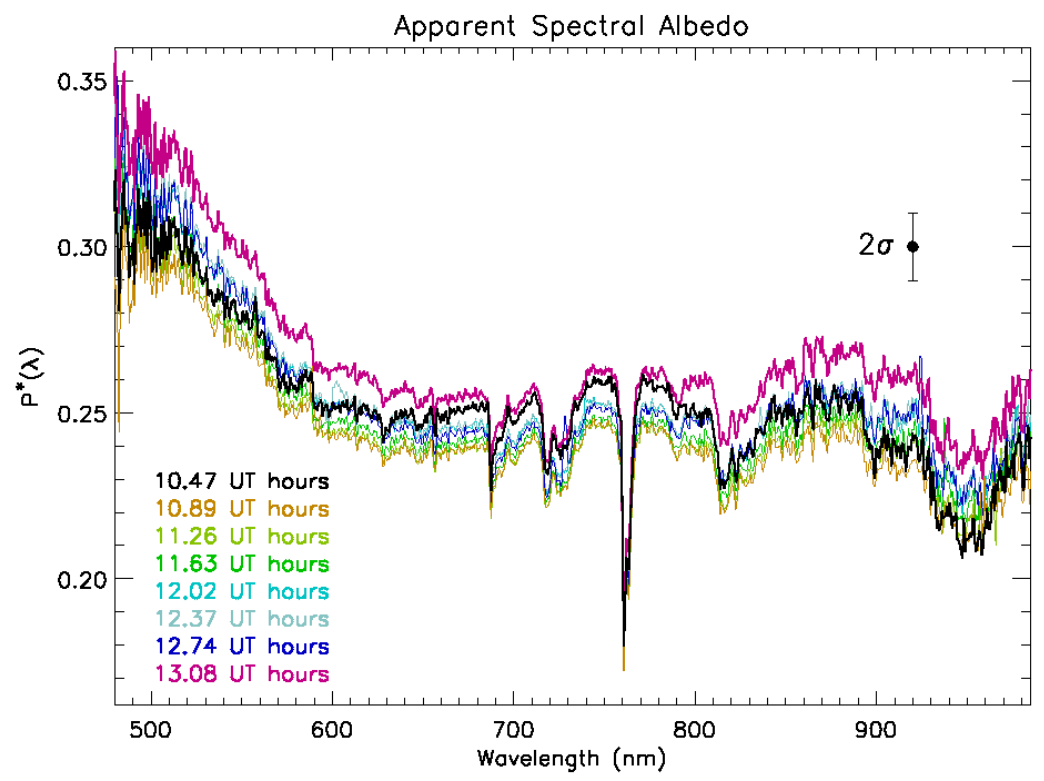

Fig. 6. Several earthshine spectra, taken with the $60^{\prime \prime}$ Telescope at Palomar Observatory in November 2003. Different colours indicate different observing times during the night. The main features of the Earth's reflectance in this region include an enhancement due to Rayleigh scattering in the blue part of the Chappuis $O_{3}$ band, which contributes to the drop above $500 \mathrm{~nm}$. Atmospheric absorption bands due to oxygen, the sharpest, $\mathrm{A}-\mathrm{O}_{2}$ at $760 \mathrm{~nm}$, and water vapour are clearly detected. Adapted from Montañés-Rodríguez et al. (2005)

through earthshine observations (Hamdani et al. 2006). In the near-infrared, the Earth's spectra is dominated by strong absorption bands of $\mathrm{H}_{2} \mathrm{O}, \mathrm{CO}_{2}$ (Turnbull et al. 2006).

Indications of a sharp increase in reflectivity near $0.720 \mu \mathrm{m}$ have also been identified as due to reflectance from vegetation (Montañés-Rodríguez et al. 2006). However, this would be an ambiguous biosignature on an exoplanet, because vegetation is not the only possible source of the reflectivity bump (Tinetti et al. 2006).

In the infrared range, earthshine observations cannot be obtained using the earthshine due to the strong absorption of the Earth's atmosphere and the emission from the Moon. Thus, observations of the infrared spectrum of Earth are only available from occasional remote spacecraft observations. The thermal emission spectrometer (TES) on the Mars Global Surveyor spacecraft acquired one such observation of the Earth from a distance of 4.7 million $\mathrm{km}$ on 1996 November 24 (Christensen \& Pearl 1997). The thermal emission of the Earth dominates the IR spectrum, corresponding to its effective temperature of $288 \mathrm{~K}$ (see Figure 7). The 


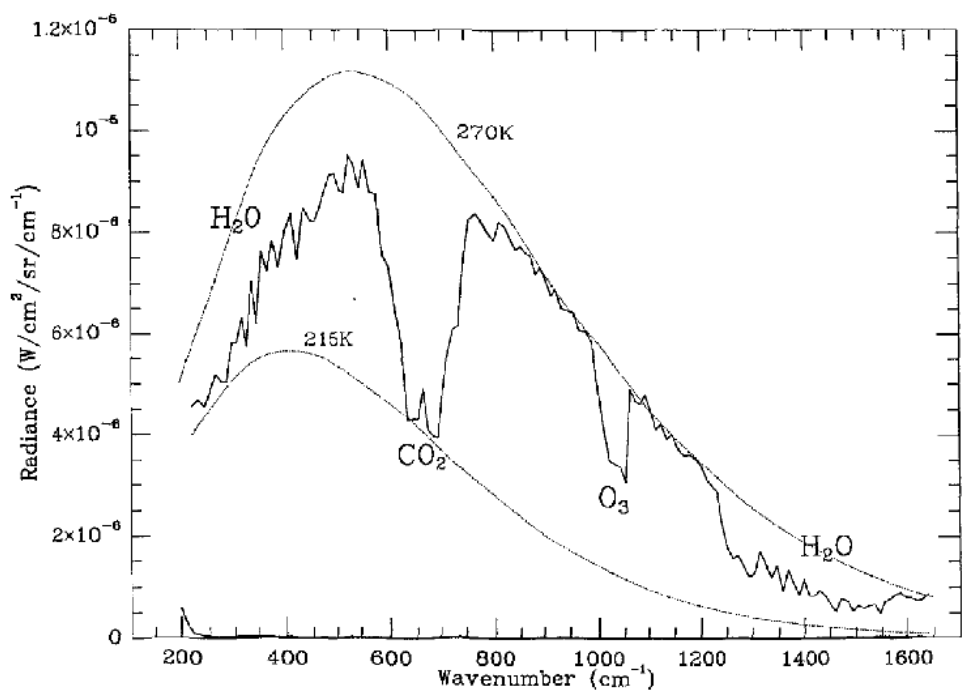

Fig. 7. Normalized calibrated spectral radiance of the Earth (central heavy curve). The data have been normalized to the highest signal. The noise equivalent spectral radiance is the lower heavy curve near the $x$ axis. Dotted curves sow blackbodies at 215 and 270 K. Adapted from Christensen \& Pearl (1997)

IR brightness peaks around $10 \mu \mathrm{m}$ and then decays slowly. Spectral features of the gases carbon dioxide $\left(\mathrm{CO}_{2}\right)$, water vapour $\left(\mathrm{H}_{2} \mathrm{O}\right)$, and ozone $\left(\mathrm{O}_{3}\right)$ dominate the Earth's spectra in this range, as well as methane $\left(\mathrm{CH}_{4}\right)$ and several other minor constituents. Radiation at the centre of the $\mathrm{CO}_{2}$ band arises mainly from the lower stratosphere; near 650 and $700 \mathrm{~cm}^{-1}$ from near the tropopause; and further into the band wings from the troposphere and surface. Thus, in the disc-averaged sense, the spectrum indicates a warm stratosphere above a tropopause somewhat colder than $215 \mathrm{~K}$.

\section{Earthshine polarization measurements}

The light emitted by the sun is unpolarized; however, when it interacts with the Earth's atmosphere it becomes polarized by transmission, reflection, refraction, or scattering (Hecht \& Zajac 1997). The degree of polarization of the globally integrated light scattered from Earth depends on the cloud coverage, together with the degree of polarization of each of the exposed areas of its surface.

Dollfus (1957) was the first to take observations of earthshine polarization and compare them to direct measurement of polarization from the ground from balloon observations. From his measurements, he concluded that the atmospheric polarization is far larger than that introduced from the ground and noted that 'for an extra-terrestrial observer it would be diluted by the intense, but little polarized, 


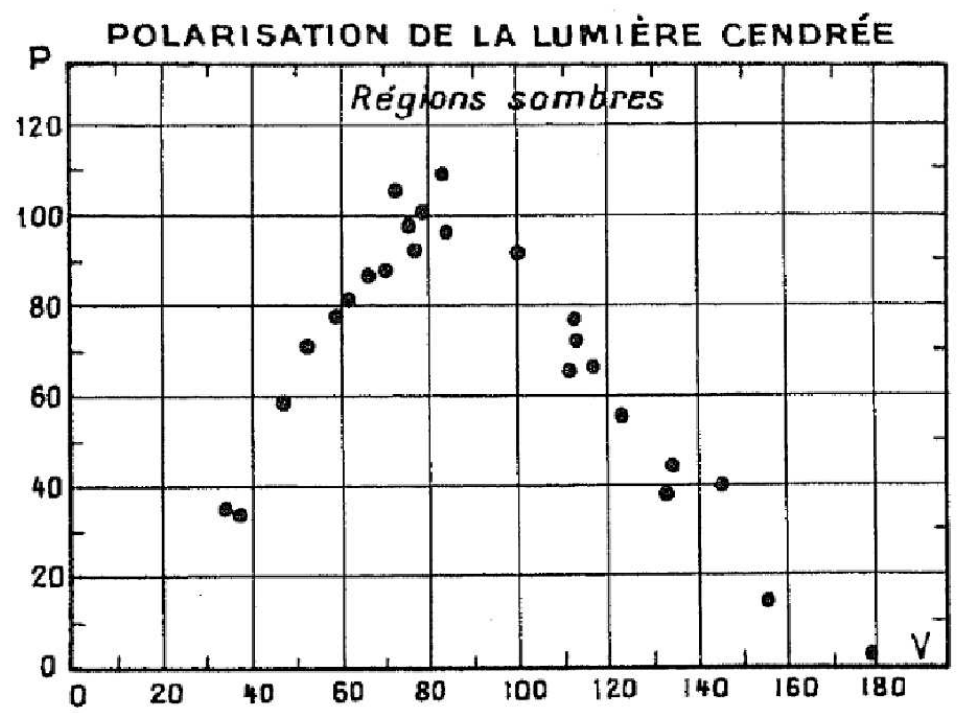

Fig. 8. The degree of polarization of the earthshine, plotted as a function of phase. These are the only existing globally-integrated measurements of the Earth's polarization, and they are only indicative, because reflection from the Moon introduces a variable depolarization factor. Adapted from Dollfus (1957).

light from the background.' He also noted how the degree of polarization was phase dependent. More recently, based on polarization observations of the Earth made from the Polder satellite, Wolstencroft \& Breon (2005) determined that the degree of linear polarization, at $443 \mathrm{~nm}$ and $90^{\circ}$ scattering angle, is $23 \%$ for averaged cloud cover conditions.

The rotation of a planet with surface features such as continents and oceans should modulate the polarized reflectances in a simple and predictable manner. The models (McCullough 2008) predict the shape of the phase function of the Earthshine's linear polarization as observed by Dollfus (see Figure 8). The maximum polarization agrees with Dollfus's observations but is approximately twice as large as that predicted by Wolstencroft \& Breon (2005). Thus, linear polarization could be a potentially useful signature of oceans and atmospheres of Earth-like extrasolar planets.

Biotic material, with its helical molecular structure, is known to produce circular polarization of reflected light in the visible range (Pospergelis 1969; Wolstencroft \& Raven 2002). Observed on the Moon, chiral signatures in the Earthshine are expected of the order of $V / I 10^{-4 \ldots-5}$, assuming a dilution of the circular polarimetric signal caused by vegetation due to partial cloud coverage (factor 10), 
and by depolarization on the lunar surface up to a factor 10 (DeBoo et al. 2005). Preliminary measurement of non-zero Stokes $V / I$ during a specific phase of the Earthshine, were observed by Sterzik et al. (2008) but could not unambiguously be interpreted as the signature of biotic homochirality, and therefore life, on Earth.

On Earth, water surfaces provide the only significant sources of thermal infrared polarized radiation, while emission from the atmosphere and ground is almost always unpolarized to any practical degree (Shaw 2002). Thus, the degree of polarization of the infrared flux emitted from Earth, observed as a planet, is negligible.

\section{ET's Conclusions}

In conclusion, if an extraterrestrial (or extrasolar) observer was looking at the Earth from an astronomical distance, and similarly if we were observing an Earthlike exoplanet, we would be able to determine some of its properties. From al these properties, those regarding the possibility of finding life would be:

- Habitability signatures:

Atmospheric composition

Variability and albedo

Spectral shape (Rayleigh scattering, atmospheric properties)

Rotational period

Presence of oceans (glint)

Presence of dynamic weather ... and possibility of liquid water

- Life signatures:

Atmospheric disequilibrium caused by life

- Complex life (plant/animal) signatures:

Presence of complex life (red or other edges)

Fluorescence

Presence of biomass from circular polarization

- Intelligent life signatures:

Direct emissions: Radio, lasers, etc.

Indirect emissions: Night lights, CFCs, ...

Unexplainable atmospheric composition / properties

Of all the signatures described above, only the habitability and life signatures are likely to be observed in extrasolar planets during the coming decades. Even then, it will require instruments and observing techniques which are nowadays only in the conceptual phase. It is extremely unlikely that we will detect any of 
the complex life signatures described in this paper, with the possible exception of the red edge, considering that exoplanets may exist in a wide range of physical properties. Even then, however, it will be very difficult to attribute the red edge to biota. The signature of intelligent life, although easier to detect from the technical point of view, requires that the alien civilization was trying to contact us, thus the author considers them very unlikely to be detected in the near future, but with an extreme desire of being proven wrong.

\section{Acknowledgements}

The contents of this paper are examined in much greater detail in the forthcoming book The Earth as a Planet, by M. Vazquez, E. Pallé and P. Montañés Rodriguez.

\section{References}

Campbell, D.B. et al. 2003, Sci, 302, 431-434

Christensen, P.R. \& Pearl, J.C. 1997, JGR, 102, 10875-10880

Deboo, B.J. et al. 2005, 44, 5434-5445

Dollfus, A. 1957, Suppl. Ann. Astroph. 4, 3-114

Hamdani, S. et al. 2006, A\&A, 460, 617-624

Hetch, E. \& Zajac, A. 1997, Optics, Addison Wesley Publishing Company; 3rd edition

Hitchcock, D.R. \& Lovelock, J.E. 1967, Icarus, 7, 149-159

Kaltenegger, L. et al. 2007, ApJ, 658, 598-616

McCullough, B. 2008, ApJ (submitted)

Montañés-Rodríguez et al. 2005, ApJ, 629, 1175-1182

Montañés-Rodríguez et al. 2006, ApJ, 651, 544-552

Pallé, E. et al. 2003, JGR, 108, 4710

Pallé, E. et al. 2004, Sci, 304, 1299-1301

Pallé, E. et al. 2008, ApJ, 676, 1319-1329

Pospergelis, M.M. 1969, SvA, 12, 973

Qiu, J. et al. 2003, JGR, 108, 4709

Rossow, W.B. et al. 1996, WMO/TD-No. 737, WMO, Geneva, 115pp.

Sagan, C. et al. 1993, Nat, 365, 715

Shaw, J.A. 2002, SPIE, 4819, 129-138

Stam, D.M. et al. 2006, A\&A,452,669-683

Sterzik, M. 2008, (in preparation)

Tinetti, G. et al. 2006, Astrobiology, 6, 881-900

Turnbull, M.C. et al. 2006, ApJ, 644, 551-559

Williams, D.M. \& Gaidos, E. 2008, ArXiv e-prints, 0801.1852

Wolstencroft, R.D. \& Breon, F.M. 2005, PASPC, 343, 211

Wolstencroft, R.D. \& Raven, J.A. 2002, Icarus, 157, 535-548

Woolf, N. J. et al. 2002, ApJ, 574, 430-433 